\begin{tabular}{|c|l|}
\hline Title & Linear Response of a V entilated Thermocline to Periodic Wind Forcing \\
\hline Author(s) & Kubokawa, A tsushi \\
\hline Citation & $\begin{array}{l}\text { Journal of Physical Oceanography, 43(8), 1811-1820 } \\
\text { https://doi.org/L.1175/PO-D-13-08.1 }\end{array}$ \\
\hline Issue Date & 2013-08 \\
\hline Doc URL & http://hdl.handle.net/2115/54713 \\
\hline Rights & ○ 2013 A merican Meteorological Society \\
\hline Type & article \\
\hline File Information & jpo-d-13-08.pdf \\
\hline
\end{tabular}

Instructions for use 


\title{
Linear Response of a Ventilated Thermocline to Periodic Wind Forcing
}

\author{
ATsushi KUBOKAWA \\ Faculty of Environmental Earth Science, Hokkaido University, Sapporo, Japan
}

(Manuscript received 30 December 2012, in final form 12 April 2013)

\begin{abstract}
This article presents a solution for the linear response of a $2 \frac{1}{2}-$-layer ventilated thermocline to large-scale periodic wind forcing, with a fixed outcrop latitude. At the eastern boundary, a Rossby wave whose vertical structure is similar to the first baroclinic mode is generated and propagates westward in the shadow zone. Meanwhile, the wave is unstable and amplified westward in the southern region. In the ventilated zone, in addition to the first-mode Rossby wave generated at the eastern boundary, two waves with second mode-like vertical structures are generated. One wave is generated directly by the wind over the outcrop. This wave has a zero zonal wavenumber and southwestward group velocity, such that the eastern edge of the wave migrates westward as it propagates southward. The other wave is generated by interaction between the westwardpropagating, first-mode Rossby wave and the outcrop. The zonal wavenumber is the same as that of the first mode at the outcrop, and the phase of the wave propagates southwestward. The crests and troughs of this wave extend across the ventilated zone from the outcrop to the internal boundary between the shadow zone and the ventilated zone.
\end{abstract}

\section{Introduction}

The oceanic response to wind forcing is an important subject with a long history of investigation in various situations (e.g., Pedlosky 1965; Anderson and Gill 1975; Anderson and Killworth 1977). In classical theories, the effects of mean flow advection are not considered. If we do not consider the bottom topography and the mean ocean circulation, the potential vorticity simply increases northward, causing the Rossby waves to propagate westward. However, in the real ocean, the potential vorticity in the thermocline is distorted from zonal uniformity because of the parcel-wise conservation of the potential vorticity (PV), as demonstrated in modern ocean circulation theories (Luyten et al. 1983; Young and Rhines 1982). According their theories, ocean gyre can be divided into three dynamically different zones: shadow, ventilated, and homogenized PV pool.

Liu (1993) and Liu and Pedlosky (1994) studied the response of a two-layer oceanic gyre consisting of these dynamically different regions to periodic forcings. However, because the two-layer model permits only one propagating

Corresponding author address: Dr. Atsushi Kubokawa, Faculty of Environmental Earth Science, Hokkaido University, Kita10 Nishi5, Kita-ku, Sapporo 060-0810, Japan.

E-mail:kubok@ees.hokudai.ac.jp mode, those studies could not represent variations of the main thermocline. To discuss wave dynamics including the variation of the main thermocline, Liu (1999a,b) used a 21/2-layer model and showed that the distorted PV distribution and the mean flow advection hardly affect the first baroclinic mode so it propagates westward, while the second mode tends to propagate along the mean PV contours of the second layer. He referred to the first baroclinic mode that is mostly unaffected by the mean current as the non-Doppler- shift mode ( $N$ mode) and to the second mode, which is mostly advected by the mean flow, as the advective mode ( $A$ mode).

Dewar and Huang (2001) investigated the adjustment of the thermocline under varying wind and buoyancy forcing over time scales from several years to decades, using a $3 \frac{1}{2}$-layer modified Hendershot model, which is a quasigeostrophic model that includes effects of ventilation from outcrops. They showed that perturbations resulting from wind stress anomalies project strongly onto the first mode, while perturbations generated by buoyancy anomalies have strong projections onto the second and third modes, propagating along pathways very close to the mean circulation, and they compared the results with results of numerical experiments based on a planetary geostrophic ocean circulation model. Kubokawa and Nagakura (2002) studied the detailed characteristics of the wave modes in a $2 \frac{1}{2} 2$-layer ventilated 
thermocline model (Luyten et al. 1983). They discussed dispersion relation, evolution of isolated initial disturbances, response to localized steady forcing, and stability in each region of the shadow zone, the ventilated zone, and the pool zone. Cerovečki and de Szoeke (2007) studied the oceanic response to periodic forcing using a $2 \frac{1}{2}$-layer model corresponding to the shadow zone of the ventilated thermocline model. Although the shadow zone is unstable with regard to longwave disturbances, Cerovečki and de Szoeke (2007) showed that the growth of the unstable wave occurs only in the southern subtropical gyre under large-scale periodic forcing.

In this brief article, we will present a linear solution for a perturbed thermocline forced by large-scale periodic wind stress using a $2 \frac{1}{2}$-layer ventilated thermocline, consisting of the shadow, the ventilated, and the pool zones. The purpose is to clarify what occurs in an idealized thermocline model when the wind is oscillating, with special attention on the role of the outcrop in generating the $A$ modes, as well as how the waves cross the boundaries between the different zones. This study is complementary to the studies mentioned above because the model used by Dewar and Huang (2001) was a modified Hendershot model, Kubokawa and Nagakura (2002) did not discuss responses to large-scale periodic forcing, and Cerovečki and de Szoeke (2007) only addressed the shadow zone.

\section{Formulation}

A spherical coordinate system is adopted in this study, with a latitude of $\theta$, a longitude of $\lambda$, and the earth's radius of $a$. The model ocean is represented by three layers: the uppermost layer is layer 1 , the middle layer is layer 2, and the third layer is assumed to be infinitely deep. We used a planetary geostrophic model consisting of the hydrostatic and geostrophic balances and mass conservation in each layer. The hydrostatic and geostrophic balances yield the following relationships among the depth of the lower interface of the $j$ th layer $h_{j}$, pressure $p_{j}$, and horizontal velocity $\mathbf{u}_{j}$ in the $j$ th layer:

$$
\begin{aligned}
p_{2} / \rho_{0} & =\gamma_{2} h_{2}, \quad p_{1} / \rho_{0}=\gamma_{2} h_{2}+\gamma_{1} h_{1}, \quad \text { and } \\
\mathbf{u}_{j} & =\frac{1}{\rho_{0} f} \mathbf{k} \times \nabla p_{j},
\end{aligned}
$$

where $f$ is the Coriolis frequency, $\rho_{j}$ is the density of the $j$ th layer, $\gamma_{j}=g\left(\rho_{j+1}-\rho_{j}\right) / \rho_{0}$ (where $g$ is the acceleration due to gravity and $\rho_{0}$ is the mean density), $\mathbf{k}$ is the unit vector directed upward, and $\nabla$ is the horizontal differential operator. Substituting Eq. (1) into the mass conservation equation for each layer yields the equations for the potential thickness (reciprocal of the potential vorticity), $q_{1}=h_{1} / f$ and $q_{2}=\left(h_{2}-h_{1}\right) / f$ :

$$
\frac{\partial q_{j}}{\partial t}+\frac{1}{\rho_{0} f} J\left(p_{j}, q_{j}\right)=-\frac{1}{f} \delta_{1 j} w_{e},
$$

where $\delta_{i j}$ is the Kronecker delta ( 1 for $i=j$ and 0 for $i \neq j$ ) and $J(*, *)$ is a Jacobian, defined as

$$
J(A, B)=\frac{1}{a^{2} \cos \theta} \frac{\partial A}{\partial \lambda} \frac{\partial B}{\partial \theta}-\frac{1}{a^{2} \cos \theta} \frac{\partial B}{\partial \lambda} \frac{\partial A}{\partial \theta} .
$$

After minor manipulations, Eq. (2) can be rewritten as

$$
\begin{aligned}
\frac{\partial h_{2}}{\partial t}-\frac{1}{2 a \cos \theta} \frac{\partial}{\partial \lambda}\left[\frac{\beta}{f^{2}}\left(\gamma_{1} h_{1}^{2}+\gamma_{2} h_{2}^{2}\right)\right] & =-w_{e} \text { and } \\
\frac{\partial}{\partial t}\left(h_{2}-h_{1}\right)+\gamma_{2} J\left(h_{2}, q_{2}\right) & =0 .
\end{aligned}
$$

Because the present study investigates the linear response of the ventilated thermocline to the periodic wind forcing, we separate the variables into the basic field and the perturbation:

$$
\begin{aligned}
w_{e} & =W_{e}+\hat{w}_{e} e^{i \sigma t}, \quad h_{j}=H_{j}+\eta_{j} e^{i \sigma t}, \\
\mathbf{u}_{j} & =\mathbf{U}_{j}+\hat{\mathbf{u}}_{j} e^{i \sigma t}, \quad q_{j}=Q_{j}+\hat{q}_{j} e^{i \sigma t}, \quad \text { and } \\
p_{j} & =P_{j}+\hat{p}_{j} e^{i \sigma t},
\end{aligned}
$$

where capital letters denote the steady basic state driven by an Ekman pumping velocity $W_{e}$ and $\sigma$ is the frequency of the periodic forcing.

\section{a. Basic state}

The basic state considered in this study is a $2^{1 / 2}-$ layer version of the ventilated thermocline model (Luyten et al. 1983), in which the second layer is assumed to outcrop along a latitude circle of $\theta=\theta_{1}$ and to have a depth, along the eastern boundary located at $\lambda=\lambda_{E}$, of a constant $H_{0}$. The steady solution of Eqs. (4) and (5) gives the basic state. It is known that the solution is divided into three different zones. $H_{1}$ and $H_{2}$ in the three zones are:

$H_{2}=H_{0} \quad$ and

$H_{1}=\left[\frac{2}{\gamma_{1}} \Phi(\lambda, \theta)\right]^{1 / 2}$ for the shadow zone,

$H_{2}=\left[\frac{2 \Phi(\lambda, \theta)+\gamma_{2} H_{0}^{2}}{\gamma_{1}\left(1-f / f_{1}\right)^{2}+\gamma_{2}}\right]^{1 / 2} \quad$ and

$H_{1}=\left(1-\frac{f}{f_{1}}\right) H_{2}$ for the ventilated zone, and 

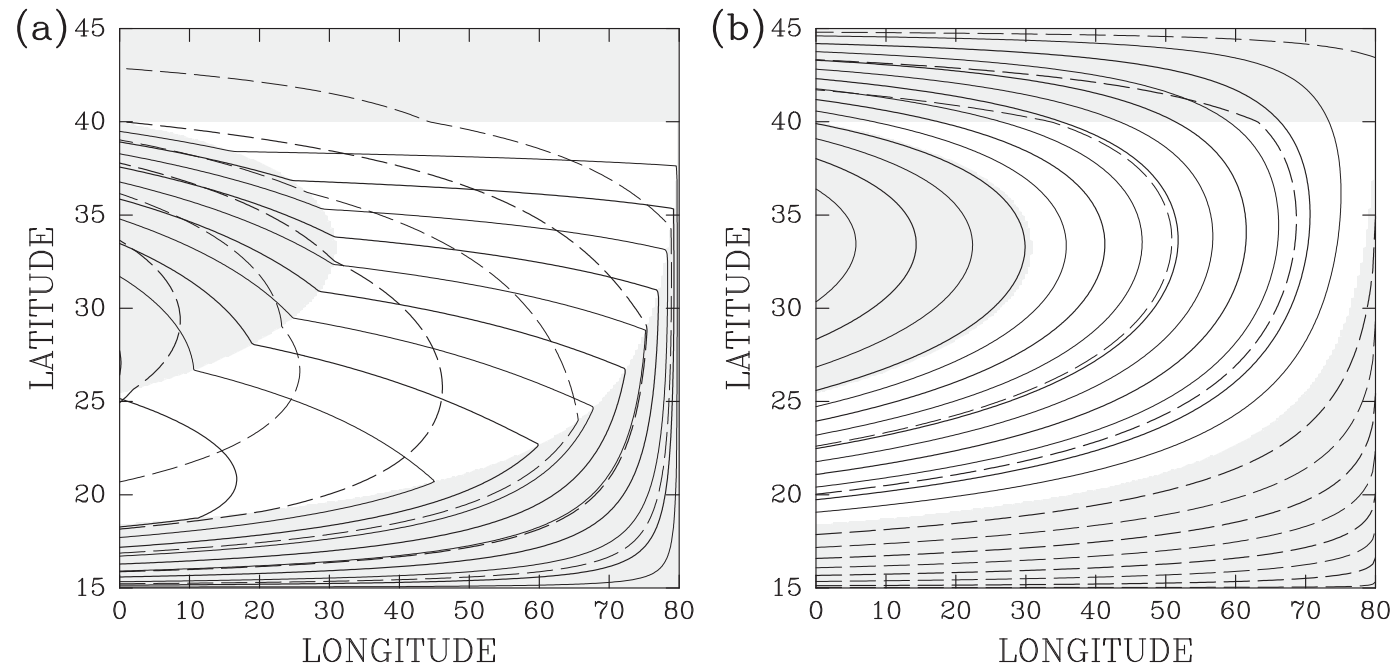

FIG. 1. Solution of the $2 \frac{1}{2}-$-layer ventilated thermocline model used as the mean state in the present study: (a) upper layer and (b) second layer. Solid contours denote layer depths $H_{1}$ and $H_{2}$ [contour interval (CI): $25 \mathrm{~m}$ ]. Dashed contours denote upper-layer geostrophic pressure, which was converted to sea surface height in (a) (CI: $0.125 \mathrm{~m})$ and potential thickness $\left(H_{2}-H_{1}\right) / f$ in $(\mathrm{b})\left(\mathrm{CI}: 1.0 \times 10^{6} \mathrm{~m} \mathrm{~s}\right)$. The shadow zone, pool zone, and the region north of the outcrop are shaded.

$$
\begin{aligned}
H_{2}= & \frac{1}{\gamma_{1}+\gamma_{2}}\left\{\gamma_{1} f Q_{2 W}+\left[\left(\gamma_{1}+\gamma_{2}\right)\left(\gamma_{2} H_{0}^{2}+2 \Phi\right)\right.\right. \\
& \left.\left.-\gamma_{1} \gamma_{2} f^{2} Q_{2 W}^{2}\right]^{1 / 2}\right\} \text { and }
\end{aligned}
$$

$H_{1}=H_{2}-f Q_{2 W}$ for the PV homogenized pool zone,

where $f_{1}=f\left(\theta_{1}\right)$ and $\Phi(\lambda, \theta)$ is the Sverdrup function. This function is defined as

$$
\Phi(\lambda, \theta)=\frac{f^{2}}{\beta} \int_{\lambda_{E}}^{\lambda} W_{e} a \cos \theta d \lambda,
$$

where $\beta=a^{-1} d f / d \theta$ and $Q_{2 W}$ is the uniform potential thickness in the potential vorticity homogenized pool (Rhines and Young 1982), which is represented as

$$
Q_{2 W}=f\left(\theta_{1}\right)^{-1}\left[H_{0}^{2}+2 \Phi\left(\lambda_{W}, \theta_{1}\right) / \gamma_{2}\right]^{1 / 2} \text {, }
$$

where $\lambda_{W}$ represents the longitude of the western boundary. In this study, the northern and southern boundaries of the subtropical gyre are $45^{\circ}$ and $15^{\circ} \mathrm{N}$, and the zonal extent is $80^{\circ}$. We chose the functional form of $W_{e}$ as

$$
W_{e}(\theta)=\frac{\beta(\theta)}{f(\theta)} \frac{f(\pi / 6)}{\beta(\pi / 6)} w_{0} \sin \left(\pi \frac{\theta-\pi / 4}{\pi / 6}\right),
$$

which yields the geostrophic Sverdrup transport having a sinusoidal form, that is,

$$
V=\frac{1}{f a \cos \theta} \frac{\partial \Phi}{\partial \lambda}=\frac{f(\pi / 6)}{\beta(\pi / 6)} w_{0} \sin \left(\pi \frac{\theta-\pi / 4}{\pi / 6}\right) .
$$

The adopted mean state is shown in Fig. 1, with $H_{0}=$ $500 \mathrm{~m}, \gamma_{1}=0.01 \mathrm{~m} \mathrm{~s}^{-2}, \gamma_{2}=0.02 \mathrm{~m} \mathrm{~s}^{-2}$, and $w_{0}=1.3 \times$ $10^{-6} \mathrm{~m} \mathrm{~s}^{-1}$.

\section{b. Equations and boundary conditions for perturbations}

Substituting Eq. (6) into Eqs. (4) and (5), and linearizing them with respect to $\eta_{1}$ and $\eta_{2}$, we obtain the equations for the perturbation:

$$
\begin{gathered}
i \sigma \eta_{2}-\frac{1}{a \cos \theta} \frac{\partial}{\partial \lambda}\left[\frac{\beta}{f^{2}}\left(\gamma_{1} H_{1} \eta_{1}+\gamma_{2} H_{2} \eta_{2}\right)\right]=-\hat{w}_{e} \text { and } \\
i \sigma\left(\eta_{2}-\eta_{1}\right)+\gamma_{2} J\left[H_{2},\left(\eta_{2}-\eta_{1}\right) / f\right]+\gamma_{2} J\left(\eta_{2}, Q_{2}\right)=0 .
\end{gathered}
$$

The assumed lateral boundary condition at the eastern boundary is $\eta_{1}=\eta_{2}=0$ and, because the outcrop latitude is fixed in this study, $\eta_{1}=0$ at $\theta=\theta_{1}$. There are two internal boundaries where $\nabla Q_{j}$ and $\mathbf{U}_{j}$ are discontinuous. The first is the boundary between the shadow zone and the ventilated zone, which is given by the curve satisfying $Q_{2}(\lambda, \theta)=H_{0} / f_{1}$, where the potential thickness of the second layer is the minimum. The other internal boundary is between the ventilated zone and the pool zone, where $H_{2}=f_{1} Q_{2 W}$. These internal boundaries are free boundaries that are advected by the perturbation 
velocity perpendicular to the boundary curve. The movement of the boundary in the direction perpendicular to the boundary curve is governed by

$$
i \sigma \xi+\mathbf{U}_{2} \cdot \nabla \xi=\mathbf{n} \cdot \hat{\mathbf{u}}_{2}=-\frac{\gamma_{2}}{f} \mathbf{k} \cdot\left(\mathbf{n} \times \nabla \eta_{2}\right),
$$

where $\xi$ is the displacement of the boundary and $\mathbf{n}$ is a horizontal unit vector perpendicular to the internal boundary $[\xi$ is defined positive when the boundary moves eastward, so $\left.\mathbf{n}=\operatorname{sgn}\left(\partial Q_{2} / \partial \lambda\right) \nabla Q_{2} /\left|\nabla Q_{2}\right|\right]$. Because the computation is carried out westward from the eastern boundary, $\eta_{2}$ on the eastern side of the internal boundary is known. Therefore, we can calculate the displacement $\xi$ by using Eq. (13). Then, $\eta_{2}$ on the western side can be calculated by Eq. (13) using the obtained $\xi$ and this $\eta_{2}$, and Eq. (12) yields $\eta_{1}$ on the western side.

\section{c. Method of solution}

Because the internal boundaries are located along the $Q_{2}$ contours, a coordinate along the $Q_{2}$ contour will be appropriate to address the problem. Therefore, we introduce $Q_{2}-y$ coordinates, where $y=a \theta$. Equations (11) and (12) become

$$
\frac{i \sigma \eta_{2}}{Q_{2 x}}-\frac{\partial}{\partial Q_{2}}\left[\frac{\beta}{f^{2}}\left(\gamma_{1} H_{1} \eta_{1}+\gamma_{2} H_{2} \eta_{2}\right)\right]=-\frac{\hat{w}_{e}}{Q_{2 x}} \quad \text { and }
$$

$\frac{i \sigma\left(\eta_{2}-\eta_{1}\right)}{\gamma_{2} Q_{2 x}}+\frac{\partial H_{2}}{\partial Q_{2}} \frac{\partial}{\partial y}\left(\frac{\eta_{2}-\eta_{1}}{f}\right)-\frac{\partial}{\partial y} \eta_{2}=0$,

where the differentials $\partial / \partial Q_{2}$ and $\partial / \partial y$ are calculated by fixing $y$ and $Q_{2}$, respectively, and $Q_{2 x}=(a \cos \theta)^{-1} \partial Q_{2} / \partial \lambda$ is the eastward gradient of $Q_{2}$. The $\partial H_{2} / \partial Q_{2}=0$ in the shadow zone and $\partial H_{2} / \partial Q_{2}=f_{1}$ in the ventilated zone. These equations cannot be applied to the pool zone because $Q_{2 x}=0$ (we will provide the equation for the pool zone at the end of this section).

The numerical method used to obtain the solution is very simple. Here, $\eta_{1}$ and $\eta_{2}$ at a grid point $(m, n)$, where $m$ and $n$ are integers increasing westward and southward, respectively, are obtained by integrating Eq. (14) from point $(m-1, n)$ to point $(m, n)$ and integrating Eq. (15) from point $(m, n-1)$ to point $(m, n)$. We used the trapezoidal formula to perform the integrations.

With regard to the matching condition at the boundary between the shadow zone and the ventilated zone, using Eq. (13), we obtain

$$
\xi=\frac{i \gamma_{2} Q_{2 x}^{(s)}}{\sigma f\left|\nabla Q_{2}^{(s)}\right|} \frac{\partial \eta_{2}^{(s)}}{\partial y} \quad \text { and }
$$

$$
\frac{\partial \eta_{2}^{(v)}}{\partial y}=\left|\nabla Q_{2}^{(v)}\right|\left[\frac{i f \sigma \xi}{\gamma_{2} Q_{2 x}^{(v)}}+f_{1} \frac{\partial \xi}{\partial y}\right],
$$

where the superscripts $(s)$ and $(v)$ denote the variables defined on the shadow zone side and the ventilated zone side, respectively, and $\nabla Q_{2}$ is the gradient of $Q_{2}$ in the $\lambda-\theta$ coordinates as in the previous sections. Equation (16) yields $\xi$ on the shadow zone side, and integrating Eq. (17) from $y=y_{1}$, where $\eta_{2}=0$, yields $\eta_{2}^{(v)}$. Here, $\eta_{1}$ on the ventilated zone side is calculated by Eq. (15) with $\eta_{1}=0$ at $y=y_{1}$.

The longitudinal displacement of the boundary between the ventilated and the pool zones at the outcrop latitude $\Delta \lambda e^{i \sigma t}$ satisfies $Q_{2}\left(\lambda_{W}+\Delta \lambda e^{i \sigma t}, \theta_{1}\right)+\hat{q}_{2} e^{i \sigma t}=Q_{2 W}$. This yields $\partial Q_{2} /\left.\partial \lambda\right|_{\lambda=\lambda_{W}} \Delta \lambda+\hat{q}_{2}\left(\lambda_{W}, y_{1}\right)=0$ at $y=y_{1}$. Therefore, $\xi$ at $y=y_{1}$ becomes $\xi=\left|Q_{2 x}^{(v)} / \nabla Q_{2}^{(v)}\right|$ $\Delta \lambda a \cos \theta_{1}=\hat{q}_{2}^{(v)} /\left|\nabla Q_{2}^{(v)}\right|$, where the superscript $(v) \mathrm{de}$ notes the variables on the ventilated zone side of the boundary. ${ }^{1}$ We can then calculate the displacement of the internal boundary $\xi$ by integrating

$$
\frac{\partial \xi}{\partial y}+\frac{i f \sigma \xi}{\gamma_{2} f_{1} Q_{2 x}^{(v)}}=\frac{1}{f_{1}\left|\nabla Q_{2}^{(v)}\right|} \frac{\partial \eta_{2}^{(v)}}{\partial y},
$$

from the outcrop $\left(y=y_{1}\right)$. Because $Q_{2}$ is uniform in the pool zone, it is appropriate to introduce $\mathrm{H}_{2}-y$ coordinates for the pool zone, with $y$-differentials calculated by fixing $H_{2}$. Then, the equation for $\eta_{2}$ along the pool zone side of the boundary becomes

$$
\frac{\partial \eta_{2}^{(p)}}{\partial y}=\left|\nabla H_{2}^{(p)}\right|\left[\frac{i f \sigma \xi}{\gamma_{2} H_{2 x}^{(p)}}+\frac{\partial \xi}{\partial y}\right]
$$

where the superscript $(p)$ denotes the variables on the pool zone side and $H_{2 x}=(a \cos \theta)^{-1} \partial H_{2} / \partial \lambda$. Integrating Eq. (19) from the outcrop where $\eta_{2}^{(p)}=\eta_{2}^{(v)}$, we obtain $\eta_{2}^{(p)}$. Using this $\eta_{2}^{(p)}$, we can calculate the disturbances in the pool zone by

$$
\frac{i \sigma \eta_{2}}{H_{2 x}}-\frac{\beta}{f^{2}} \frac{\partial}{\partial H_{2}}\left[\left(\gamma_{1} H_{1}+\gamma_{2} H_{2}\right) \eta_{2}\right]=-\frac{\hat{w}_{e}}{H_{2 x}} .
$$

Although this equation can be solved analytically, we solved it numerically.

\footnotetext{
${ }^{1}$ The longitudinal oscillation of the boundary, which is defined by $q_{2}=Q_{2 W}$, at the outcrop latitude would cause ventilation of fluid with slightly different potential thickness into the pool zone, because the second-layer depth at $\left(\lambda_{W}, \theta_{1}\right)$ also oscillates. However, because the second-layer potential thickness anomalies are only advected by the mean flow in the pool zone and this is a linear theory, we do not need to consider this small anomaly confined to a very narrow band along the boundary.
} 

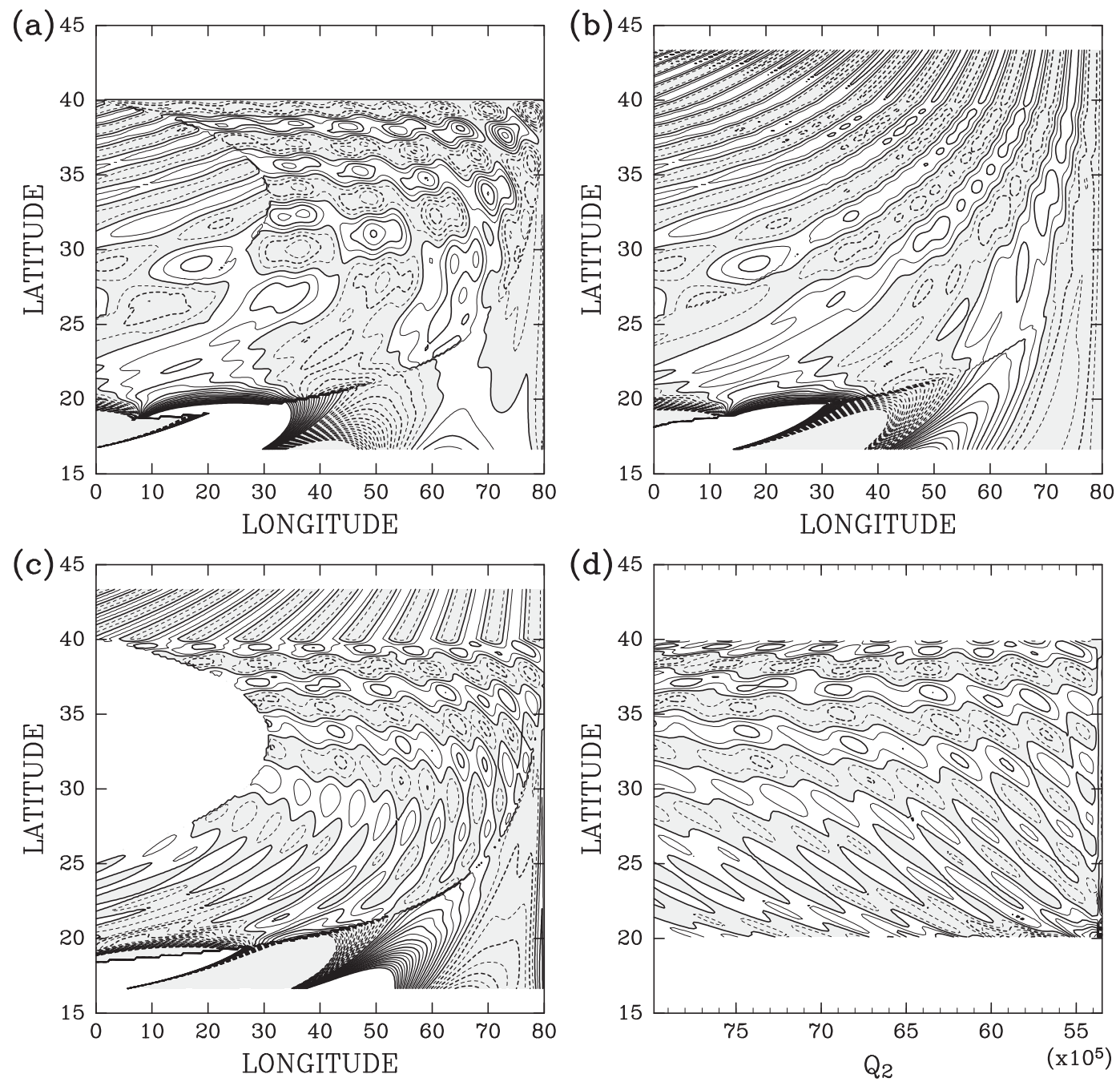

FIG. 2. Real parts of the wave solution forced by a large-scale oscillating wind over a 2-yr period: (a) $\eta_{1}(\lambda, \theta)$, (b) $\eta_{2}(\lambda, \theta)$, (c) potential thickness of the second layer $\hat{q}_{2}(\lambda, \theta)$, and (d) $\hat{q}_{2}$ on the $Q_{2}-y$ plane for the ventilated zone $\hat{q}_{2}\left(Q_{2}, \theta\right)$. The dotted contours denote the negative values. The CI is $5 \times \hat{w}_{e} / w_{0} \mathrm{~m}$ for $\eta_{1}$ and $\eta_{2}$, and is $8 \times 10^{4} \times \hat{w}_{e} / w_{0} \mathrm{~ms}$ for $\hat{q}_{2}$. The shading denotes negative values and regions where the absolute value is too large $(>20 \times \mathrm{CI})$ are not contoured.

\section{Results}

The real parts of the interface displacements $\eta_{1}$ and $\eta_{2}$, as well as the second-layer potential thickness anomaly $\hat{q}_{2}$, are shown in Fig. 2 for the case of a 2 -yr forcing period (730 days) and spatially uniform $\hat{w}_{e}$. The patterns of the imaginary parts are nearly the same, with the exception that the phase shifts by $\pi / 2$ in the opposite direction of the wave propagations and the spatially uniform deepening of the interfaces directly caused by $\hat{w}_{e}$. The results are qualitatively independent of the period and differences occur in the amplitude and wavelength, which are proportional to the period. Although the forcing amplitude in the real ocean is largest for a 1-yr period, we show a 2-yr-period solution because the wavelengths of the solutions for the 1-yr-period forcing are too short to produce clear figures.

In the shadow zone, the wave is unstable in the southern part and is amplified westward where the phase difference between $\eta_{2}$ and $\eta_{1}$ is optimal for baroclinic instability, while it is neutral in the northern part. Because this is a longwave theory, the smaller the wavelength is, the larger the growth rate is. However, the large-scale wind forcing generates the large-scale disturbance, and, therefore, the growth of the wave is modest, as discussed by Cerovečki and de Szoeke (2007).

The internal boundary between the shadow zone and the ventilated zone does not affect the wave structure in 

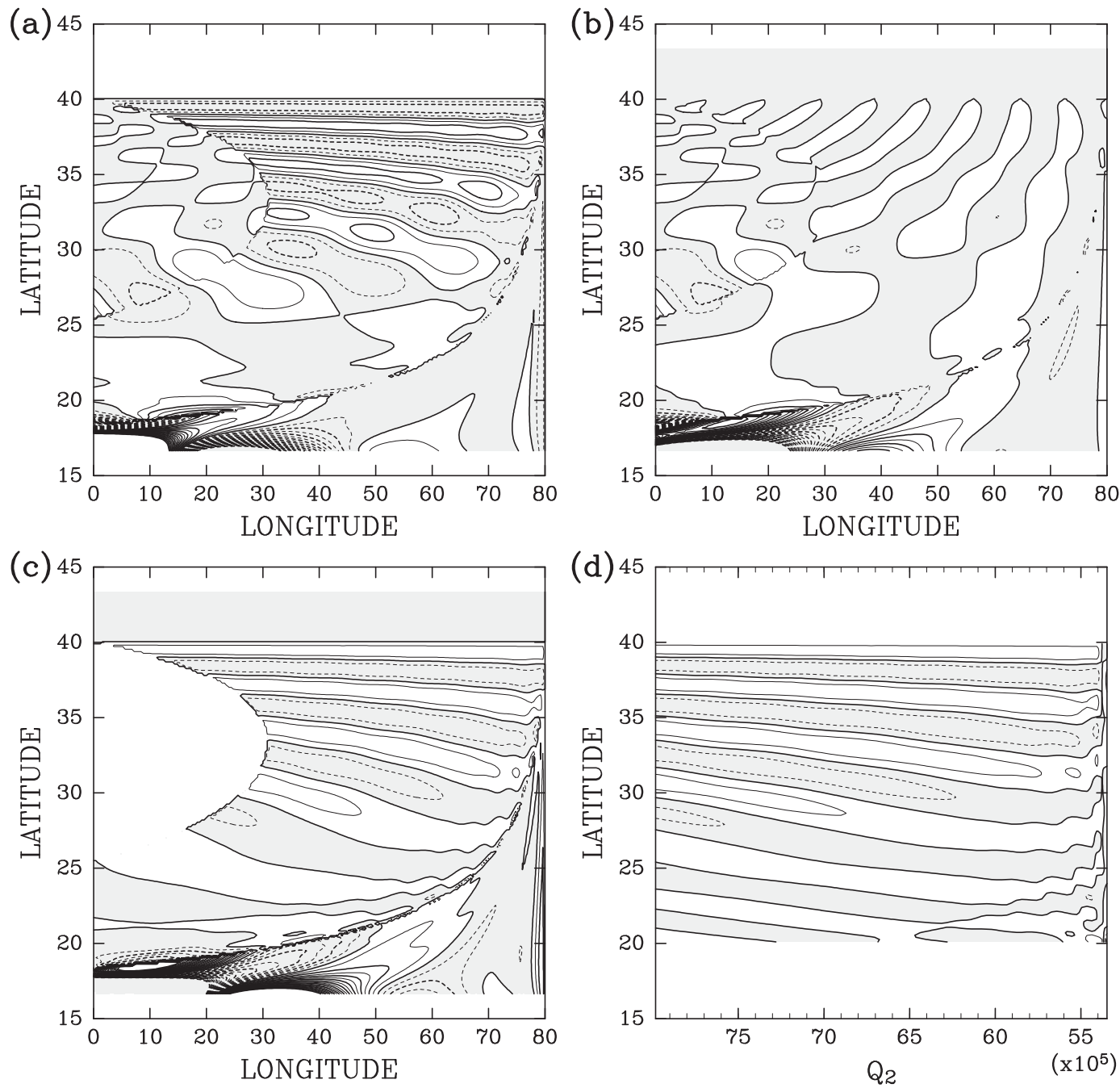

FIG. 3. As in Fig. 2, but for wave components generated by open ocean wind. In this solution, the effect of the eastern boundary is eliminated.

$\eta_{2}$ significantly, and the wave propagates into the ventilated zone beyond it. However, the large-scale structure seen in $\hat{q}_{2}$ in the shadow zone seems to be blocked by the internal boundary. Because $\left|Q_{2 x}\right|$ in the ventilated zone is much smaller than that in the shadow zone, the oscillation of $\xi$ does not cause significant changes in $\hat{q}_{2}$ in the ventilated zone; therefore, the large-scale $\hat{q}_{2}$ pattern seen in the shadow zone hardly crosses this internal boundary.

In the ventilated zone, the patterns seen in $\eta_{1}$ and $\hat{q}_{2}$ are complicated. It seems that zonal structures dominate in the northern half of the ventilated zone, while shortwave signals whose wavenumbers are directed southeastward in the $x-y$ plane (southwestward in the $Q_{2}-y$ plane) dominate in the southern half. Here, we separate the disturbances into the waves excited at the eastern boundary and those excited by Ekman pumping in the interior ocean. The results are shown in Figs. 3 and 4.

Figure 3 shows the wave field when the effect of the eastern boundary is eliminated. To eliminate the effect of the eastern boundary, we set $\eta_{1}=\eta_{2}=i \hat{w}_{e} / \sigma$ at the eastern boundary. Under this condition, the zonal gradients in $\eta_{1}$ and $\eta_{2}$ are kept vanishing at the eastern boundary under the periodic wind forcing. This figure shows that the outcrop is a wave source of the zonal $A$ mode. As it propagates southward, the eastern edge of the $A$ mode migrates westward, and the wavenumber vector rotates slightly clockwise. A theoretical interpretation of this $A$ mode will be given in the next section. The unstable growth of the wave in the southern region of the shadow zone can also be seen. Because the stratification and mean flow depend on the location, spatially uniform perturbed Ekman pumping can generate waves 

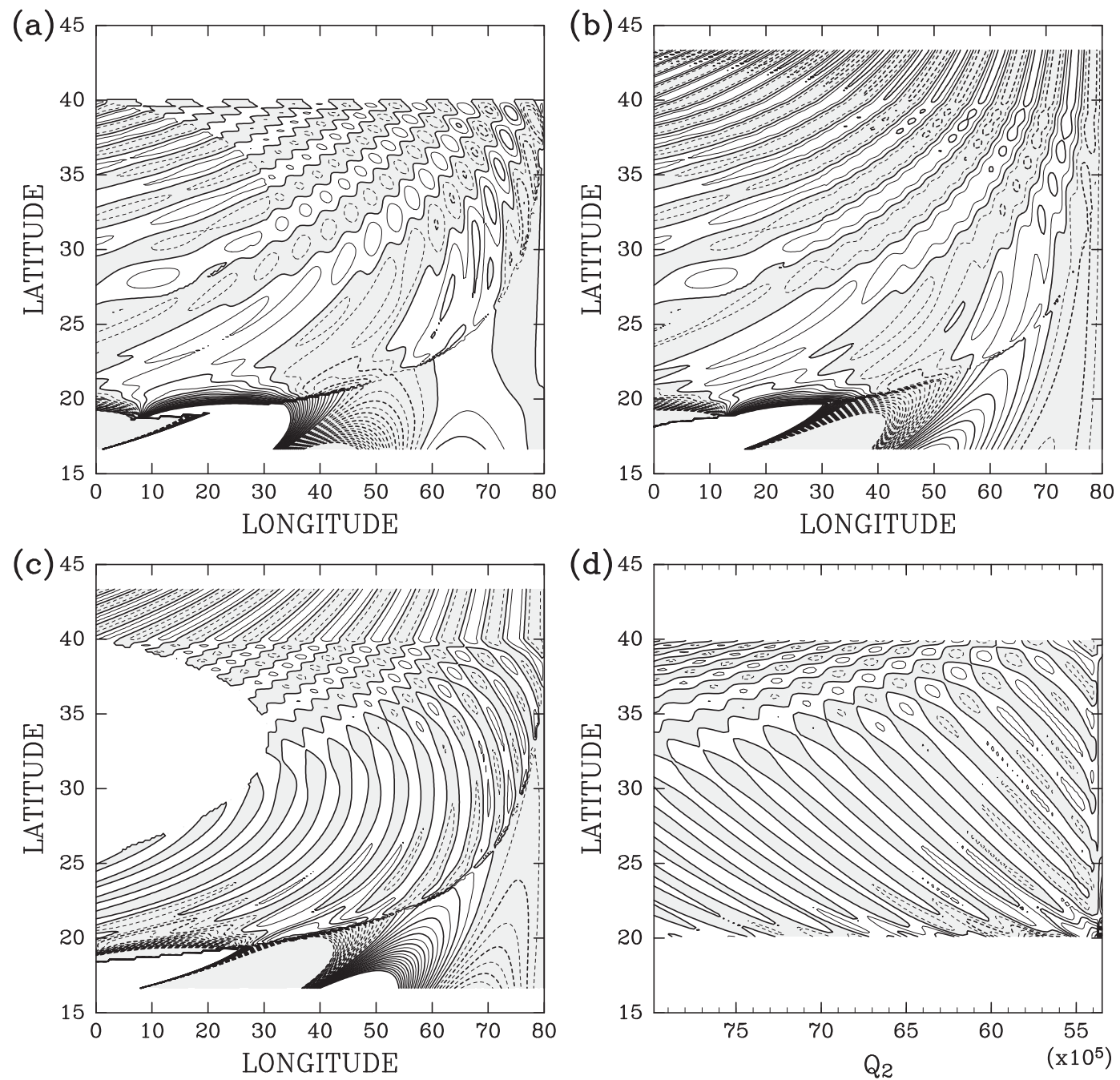

FIG. 4. As in Fig. 2, but for wave components generated at the eastern boundary.

in the interior region, although they are not significant except for the $A$ mode excited at the outcrop and the unstable wave in the shadow zone.

Figure 4 shows waves generated at the eastern boundary, where $\eta_{1}=\eta_{2}=-i \hat{w}_{e} / \sigma$ and the perturbed Ekman pumping is set to zero. The distribution of $\eta_{2}$ in this case is very similar to that in Fig. 2, while $\eta_{1}$ and $\hat{q}_{2}$ in the ventilated zone are dominated by a short wave whose wavenumber is directed southwestward in the $Q_{2}-y$ plane (Fig. $4 \mathrm{~d}$ ). This $A$-mode signal is generated at the outcrop associated with westward propagation of the $N$ mode and will be discussed in the next section.

Although there is no $A$ mode in the pool zone, Fig. 3 shows that the zonal $A$ mode generated at the outcrop by wind generates an $N$ mode in the pool zone, which complicates the distribution of $\eta_{2}$ in the pool zone. This can be interpreted as follows: the oscillating $w_{e}$ causes spatially uniform change in $\eta_{2}$, which oscillates the boundary between the ventilated and the pool zones (i.e., $\xi$ ) at the outcrop latitude. This oscillation in $\xi$ does not accompany any wavy $\eta_{2}$ on the ventilated zone side and propagates southward satisfying

$$
i \sigma \xi+\mathbf{U}_{2} \cdot \nabla \xi=0
$$

On the pool zone side, however, the wavy $\xi$ will yield $\mathbf{n} \cdot \hat{\mathbf{u}}_{2}$ in Eq. (13), because the advection velocity $\mathbf{U}_{2}$ is different between the ventilated and pool zones. Because the southward component of $\mathbf{U}_{2}$ on the ventilated zone side is the same as the southward phase speed of the zonal $A$ mode as will be shown in the next section, the zonal $A$ mode in the ventilated zone appears to generate the $N$ mode in the pool zone. 
$(\times 0.01 / \mathrm{km})$

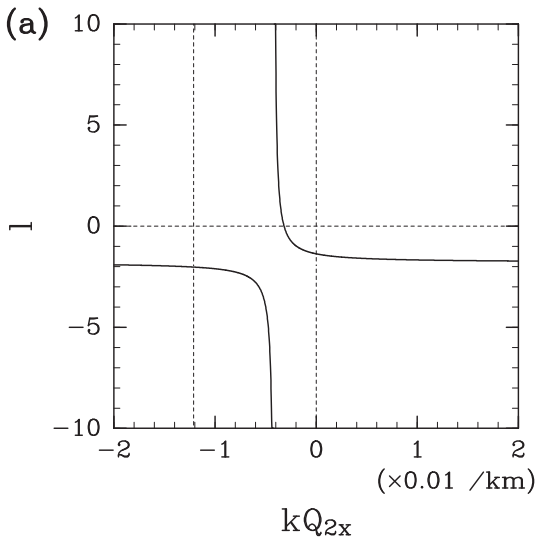

$(\times 0.01 \mathrm{~m} / \mathrm{s})$

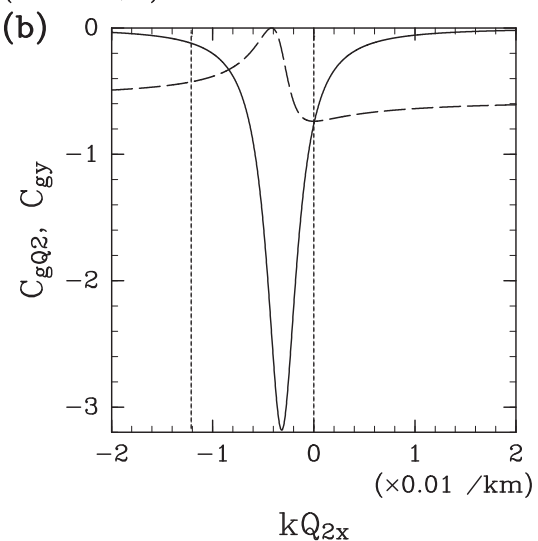

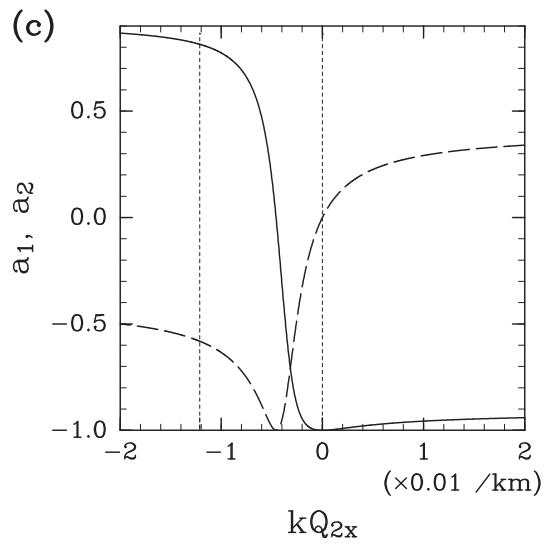

FIG. 5. Properties of a wave based on a 2 -yr period at $\left(30^{\circ} \mathrm{N}, 60^{\circ} \mathrm{E}\right)$ in the ventilated zone: (a) dispersion relation $(k-l$ relation), (b) group velocity (solid and dashed curves denote the zonal and meridional components, respectively), and (c) vertical structure (solid and dashed curves denote the amplitudes of $\eta_{1}$ and $\eta_{2}$, respectively, and are normalized as $\left|\eta_{1}\right|^{2}+\left|\eta_{2}\right|^{2}=1$ ). The wavenumber vector on the $Q_{2}-y$ plane is $(k, l)$. Here, $k$ has the same dimension as $Q_{2}^{-1}$, so we used $k Q_{2 x}$ to change the dimension to be per length. The group velocity is also defined on the $Q_{2}-y$ plane, and $c_{g Q_{2}}=\partial \sigma / \partial k Q_{2 x}^{-1}$. The thin, vertical dotted line around $k Q_{2 x}=-1.2 \times 10^{-2} \mathrm{~km}^{-1}$ denotes the wavenumber of the $N$ mode generated at the eastern boundary of the outcrop latitude.

\section{Wave generation and propagation in the ventilated zone}

If we assume that the wavelength is much shorter than the basin scale, Eqs. (14) and (15) can be rewritten as

$$
i \sigma \eta_{2}-\frac{\beta Q_{2 x}}{f^{2}}\left(\gamma_{1} H_{1} \frac{\partial \eta_{1}}{\partial Q_{2}}+\gamma_{2} H_{2} \frac{\partial \eta_{2}}{\partial Q_{2}}\right)=-\hat{w}_{e} \quad \text { and }
$$

$i \sigma\left(\eta_{2}-\eta_{1}\right)+\gamma_{2} Q_{2 x}\left[\frac{f_{1}}{f} \frac{\partial}{\partial y}\left(\eta_{2}-\eta_{1}\right)-\frac{\partial \eta_{2}}{\partial y}\right]=0$.

Substituting $\left(\eta_{1}, \eta_{2}\right)=\left(a_{1}, a_{2}\right) e^{-i\left(k Q_{2}+l y\right)}$ into Eqs. (21) and (22) and setting $\hat{w}_{e}=0$ gives the dispersion relation:

$$
\left|\begin{array}{cc}
\frac{\beta \gamma_{1} H_{1}}{f^{2}} Q_{2 x} k & \sigma+\frac{\beta \gamma_{2} H_{2}}{f^{2}} Q_{2 x} k \\
-\sigma+\frac{f_{1} \gamma_{2} Q_{2 x}}{f} l & \sigma+\frac{\gamma_{2} Q_{2 x}\left(f-f_{1}\right)}{f} l
\end{array}\right|=0 .
$$

From this equation, we can easily obtain $l=l(k, \sigma)$, group velocity in the $Q_{2}-y$ plane, $(\partial \sigma / \partial k, \partial \sigma / \partial l)$, and the vertical structure $\left(a_{1}, a_{2}\right)$, which is obtained by substituting $l=l(k, \sigma)$ into Eqs. (21) or (22). These wave properties are depicted in Fig. 5 for the 2-yr period at $30^{\circ} \mathrm{N}$ and $60^{\circ} \mathrm{E}$. These wave properties in the $Q_{2}-y$ plane are qualitatively independent of the latitude. The wave with a first mode-like vertical structure (i.e., $N$ mode) has the eastward component of wavenumber around $k Q_{2 x} \simeq$ $-0.4 \times 10^{-2} \mathrm{~km}^{-1}$, and this wave dominates the variation in $\eta_{2}$ in Figs. 2 and 4. When $l=0, \hat{q}_{2}=\left(\eta_{2}-\eta_{1}\right) / f$ is zero, while $\hat{q}_{2} \neq 0$ for $l \neq 0$ as seen in Fig. $5 \mathrm{~b}$. North of the outcrop latitude $\left(y>y_{1}\right)$, there is only one vertical mode propagating westward at the speed of $\beta f^{-2} \gamma_{2} \mathrm{H}_{2}$ that is independent of $l$ and $k=-\sigma f^{2}\left(\beta \gamma_{2} H_{2} Q_{2 x}\right)^{-1}$. The eastward component of the wavenumber $k$ of this mode at the eastern boundary of the outcrop latitude multiplied by $Q_{2 x}$ at $30^{\circ} \mathrm{N}$ and $60^{\circ} \mathrm{E}$ is $-1.2 \times 10^{-2} \mathrm{~km}^{-1}$ and is denoted by thin, vertical dotted lines in Fig. 5. At the outcrop latitude, the $N$ mode generates an $A$ mode with this zonal wavenumber. The wave with the southwestward wavenumber vector seen in $\eta_{1}$ and $\hat{q}_{2}$ in Fig. 4 is this $A$ mode. Conversely, the wave with a nearly zero zonal wavenumber dominates the variation in $\eta_{1}$ and $\hat{q}_{2}$ in Fig. 3.

Here, we discuss the generation mechanisms of these $A$-mode structure waves. First, we consider the generation of the zonal mode at the outcrop resulting from the wind variation. If there is no $N$ mode, Eq. (21) yields $\eta_{2}=i \hat{w}_{e} / \sigma$. Substituting $\eta_{2}=i \hat{w}_{e} / \sigma$ into Eq. (22) yields

$$
\frac{\partial \eta_{1}}{\partial y}+i l_{A 1}\left(\eta_{1}-\frac{i \hat{w}_{e}}{\sigma}\right)=0,
$$

where $l_{A 1}=f \sigma / f_{1} \gamma_{2} Q_{2 x}$, and the solution becomes

$$
\eta_{1}=\frac{i \hat{w}_{e}}{\sigma}\left(1-e^{-i \int_{y_{1}}^{y} l_{A 1} d y}\right),
$$

where the boundary condition $\eta_{1}\left(y_{1}\right)=0$ is used. The meridional wavenumber $l_{A 1}$ is $l$ at $k=0$. In the east $l_{A 1}$ is 
smaller because $Q_{2 x}$ is larger in the east. This result explains why the wavenumber vector rotates slightly clockwise to the south. It should also be noted that the phase speed, $\sigma / l_{A 1}=f^{-1} \gamma_{2} H_{2 x}$, is the same as the advection velocity. The direction of the group velocity of this mode is southwestward, as shown in Fig. 5b, such that the eastern edge of this mode migrates westward and exits from the ventilated zone around the central latitude of the subtropical gyre.

The generation mechanism of the $A$ mode in the boundary-forced case is more complicated than that of the wind-forced $A$ mode. At the limit of $y \rightarrow y_{1}$, because $H_{1} / H_{2} \ll 1$ and $f_{1} / f-1 \ll 1$ for $y \simeq y_{1}$, Eqs. (21) and (22) become

$i k_{N} \eta_{2}+\frac{\partial \eta_{2}}{\partial Q_{2}}=0 \quad$ and $\quad i l_{A 2}\left(\eta_{2}-\eta_{1}\right)-\frac{\partial \eta_{1}}{\partial y}=0$,

where $k_{N}=-\sigma f_{1}^{2} / \gamma_{2} \beta H_{2} Q_{2 x}$ and $l_{A 2}=\sigma / \gamma_{2} Q_{2 x}$. The $\mathrm{N}$-mode solution of these equations is solved with the zonal wavenumber $k_{N}$, whose vertical structure depends on the meridional wavenumber $l$ as $a_{1} / a_{2}=l_{A 2} /\left(l_{A 2}-l\right)$. The $A$ mode in Eq. (26) has $\eta_{2}=0$ and a meridional wavenumber of $l_{A 2}$; the zonal wavenumber is arbitrary. The boundary condition $\eta_{1}=\eta_{2}=-i \hat{w}_{e} / \sigma$ at $Q_{2}=$ $Q_{2 E}=H_{0} / f_{1}$ (the eastern boundary of the ventilated zone) generates the $N$ mode with $l=0$ and $\eta_{1}=\eta_{2}=$ $-i \hat{w}_{e} / \sigma$. On the other hand, because $\eta_{1}=0$ at $y=y_{1}$ must be satisfied for $Q_{2}>Q_{2 E}$, an $A$ mode with the amplitude of $i \hat{w}_{e} / \sigma$ is generated. Therefore, the solution becomes

$$
\begin{aligned}
& \eta_{2}=-\frac{i \hat{w}_{e}}{\sigma} e^{-i \int_{Q_{2 E}}^{Q_{2}} k_{N} d Q_{2}} \text { and } \\
& \eta_{1}=\frac{i \hat{w}_{e}}{\sigma} e^{-i \int_{Q_{2 E}}^{Q_{2}} k_{N} d Q_{2}}\left[e^{-i l_{A 2}\left(y-y_{1}\right)}-1\right] \text { for } Q_{2}>Q_{2 E} .
\end{aligned}
$$

The amplitude of the $A$ mode abruptly changes from 0 at the boundary between the shadow zone and ventilated zone $\left(Q_{2}=Q_{2 E}\right)$ to $\hat{w}_{e} / \sigma$ for $Q_{2}>Q_{2 E}$. Because an $A$ mode with a short zonal wavelength propagates along the $Q_{2}$ contour (i.e., $\partial \sigma / \partial k=0$; see Fig. $5 \mathrm{~b}$ ), the $A$ mode generated by the $N$ mode at the outcrop line is trapped at and propagates along the internal boundary of $Q_{2}=$ $Q_{2 E}$. As a result, the $A$ mode of the zonal wavenumber of $k_{N}\left(y_{1}\right)$ seems to act as a bridge connecting the $N$ mode propagating westward along the outcrop to the $A$ mode propagating southward along the internal boundary. A similar wave solution structure was reported by Kubokawa and Nagakura (2002) for the initial value problem. It should be noted that $|l|$ of the $N$ mode increases westward because of the meridional gradient of the westward phase speed. Because of the $\hat{q}_{2}$ anomaly associated with the $N$ mode when $l \neq 0$, the $\hat{q}_{2}$ anomaly in Figs. $4 \mathrm{c}$ and $4 \mathrm{~d}$ exhibits a diamond-like checkerboard pattern caused by the superposition of the $N$ and $A$ modes in the northwestern ventilated zone, where the meridional wavenumber of the $N$ mode is large.

\section{Summary}

In this article, a linear longwave solution in a $2 \frac{1}{2}$-layer ventilated thermocline responding to periodic wind forcing was obtained in the case that the density boundary conditions (interface depths at the eastern boundary and outcrop latitude) are fixed. In the shadow zone, there are unstable waves whose amplitude increases westward, as discussed by Cerovečki and de Szoeke (2007). In the ventilated zone, in addition to the $N$ mode (the first baroclinic mode) generated at the eastern boundary, the $A$ modes (higher baroclinic modes) are generated at the outcrop. One $A$ mode is generated by the interaction between the westward-propagating $N$ mode and the outcrop, whose zonal wavenumber is the same as that of the $N$ mode at the outcrop. The other $A$ mode is generated by the wind over the outcrop whose zonal wavenumber is zero. Because the group velocity of this mode is southwestward, its eastern edge migrates westward, and because the southward speed is higher in the east, the wavenumber vector rotates slightly clockwise as it propagates southward.

It has been thought that $A$ modes are mainly excited by thermal forcing. In addition to the thermal forcing, the present study suggests that wind forcing around the outcrop and westward propagation of the $N$ mode can also significantly excite $A$ modes. If there is no outcrop, the direct wind forcing tends to move all the interfaces in the same vertical direction, so that only $N$ modes are excited. However, in the present situation, because the outcrop latitude is fixed independently of the wind, the potential thickness in the second layer changes at the outcrop if $\eta_{2}$ changes, and this potential thickness anomaly is advected and propagates southward as the $A$ mode. A similar mechanism works when the $N$ mode propagates along the outcrop. In the real ocean, surface thermal forcing is important as well, which changes the outcrop latitude. The oscillation of the outcrop latitude generates $A$ mode disturbances. Under realistic forcing, both effects are important.

Acknowledgments. The author thanks anonymous reviewers for helpful comments. This study was partially supported by Grant-in-Aid for Scientific Research from MEXT, Japan, and JSPS (21540447, 22106006, and 
23340139). The GFD DENNOU library was used for drawing the figures.

\section{REFERENCES}

Anderson, D. L. T., and A. E. Gill, 1975: Spin-up of a stratified ocean, with applications to upwelling. Deep-Sea Res., 22, 583 596.

, and P. D. Killworth, 1977: Spin-up of a stratified ocean, with topography. Deep-Sea Res., 24, 709-732.

Cerovečki, I., and R. A. de Szoeke, 2007: How purely wind-driven long planetary geostrophic waves may be energized in the western part of ocean subtropical gyres. J. Phys. Oceanogr., 37, $60-70$.

Dewar, W. R., and R. X. Huang, 2001: Adjustment of the ventilated thermocline. J. Phys. Oceanogr., 31, 1676-1694.

Kubokawa, A., and M. Nagakura, 2002: Linear planetary wave dynamics in a 2.5-layer ventilated thermocline model. J. Mar. Res., 60, 367-404.
Liu, Z., 1993: Thermocline forced by varying Ekman pumping. Part II: Annual and decadal Ekman pumping. J. Phys. Oceanogr., 23, 2523-2540.

_ 1999a: Forced planetary response in a thermocline gyre. J. Phys. Oceanogr., 29, 1036-1055.

- 1999b: Planetary wave modes in the thermocline: Nondoppler-shift mode, advective mode and Green mode. Quart. J. Roy. Meteor. Soc., 125, 1315-1339.

— , and J. Pedlosky, 1994: Thermocline forced by annual and decadal surface temperature variation. J. Phys. Oceanogr., 24, 587-608.

Luyten, J., J. Pedlosky, and H. Stommel, 1983: The ventilated thermocline. J. Phys. Oceanogr., 13, 293-309.

Pedlosky, J., 1965: A study of the time-dependent ocean circulation. J. Atmos. Sci., 22, 267-272.

Rhines, P. B., and W. R. Young, 1982: A theory of the wind-driven circulation. I. Mid-ocean gyres. J. Mar. Res., 40 (Suppl.), $559-596$.

Young, W. R., and P. B. Rhines, 1982: A theory of the wind-driven circulation. II. Gyres with western boundary layers. J. Mar. Res., 40 (Suppl.), 849-872. 